\title{
Boron-Doped Reduced Graphene Oxide with Tunable Bandgap and Enhanced Surface Plasmon Resonance
}

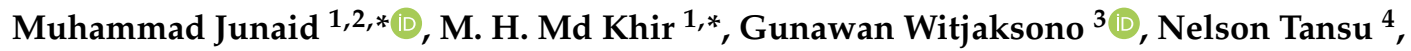 \\ Mohamed Shuaib Mohamed Saheed ${ }^{5}(0)$, Pradeep Kumar ${ }^{1}\left(\mathbb{D}\right.$, Zaka Ullah $\left.{ }^{1, *} \mathbb{(}\right)$, Asfand Yar 6 \\ and Fahad Usman ${ }^{6}$ \\ 1 Department of Electrical and Electronic Engineering, Universiti Teknologi PETRONAS, Seri Iskandar 32610, \\ Perak, Malaysia; Pradeep.hitesh@gmail.com \\ 2 Department of Electronic Engineering, Balochistan University of Information Technology, Engineering, \\ and Management Sciences, Quetta 87300, Balochistan, Pakistan \\ 3 BRI Institute, Jl. Harsono RM No.2, Ragunan, Passsar Minggu, Jakarta 12550, Indonesia; \\ Gunawan.witjaksono@gmail.com \\ 4 Center for Photonics and Nanoelectronics, Department of Electrical and Computer Engineering, \\ Lehigh University, 7 Asa Drive, Bethlehem, PA 18015, USA; Tansu@lehigh.Edu \\ 5 Department of Mechanical Engineering, Universiti Teknologi PETRONAS, Seri Iskandar 32610, Perak, \\ Malaysia; Shuaib.saheed@utp.edu.my \\ 6 Department of Fundamental and Applied Sciences, Universiti Teknologi PETRONAS, Seri Iskandar 32610, \\ Perak, Malaysia; Asfandyarhargan@gmail.com (A.Y.); Fahad_17004459@utp.edu.my (F.U.) \\ * Correspondence: Muhammad_17000796@utp.edu.my (M.J.); Harisk@utp.edu.my (M.H.M.K.); \\ Zaka_18000817@utp.edu.my (Z.U.)
}

Academic Editor: Daniela Meroni

Received: 10 June 2020; Accepted: 30 June 2020; Published: 11 August 2020

\begin{abstract}
Graphene and its hybrids are being employed as potential materials in light-sensing devices due to their high optical and electronic properties. However, the absence of a bandgap in graphene limits the realization of devices with high performance. In this work, a boron-doped reduced graphene oxide (B-rGO) is proposed to overcome the above problems. Boron doping enhances the conductivity of graphene oxide and creates several defect sites during the reduction process, which can play a vital role in achieving high-sensing performance of light-sensing devices. Initially, the B-rGO is synthesized using a modified microwave-assisted hydrothermal method and later analyzed using standard FESEM, FTIR, XPS, Raman, and XRD techniques. The content of boron in doped rGO was found to be 6.51 at.\%. The B-rGO showed a tunable optical bandgap from 2.91 to $3.05 \mathrm{eV}$ in the visible spectrum with an electrical conductivity of $0.816 \mathrm{~S} / \mathrm{cm}$. The optical constants obtained from UV-Vis absorption spectra suggested an enhanced surface plasmon resonance (SPR) response for B-rGO in the theoretical study, which was further verified by experimental investigations. The B-rGO with tunable bandgap and enhanced SPR could open up the solution for future high-performance optoelectronic and sensing applications.
\end{abstract}

Keywords: graphene oxide; microwave; hydrothermal; boron-doped reduced graphene oxide; oxygen reduction reaction; optical bandgap; surface plasmons resonance

\section{Introduction}

Surface plasmon resonance (SPR) biosensors are optical sensors that have attracted greater attention due to their advantages such as high sensitivity, room temperature real-time measurements, and non-invasive measurements [1]. SPR is extremely sensitive to the alteration of the refractive index at the metal-dielectric interfac [2]. As such, sensitivity is exploited in the detection by any change at the interface due to the presence of an analyte. Gold is typically used as a resonant layer in SPR 
biosensors due to its chemical stability, where gold exhibits a higher shift of resonant angle with the change in refractive index [3]. However, the inertness of gold makes the detection or adsorption of the biomolecules very difficult at low concentrations, and sometimes even impossible [4-6]. Specifically, the single-crystalline gold surface does not measure the adsorption of small molecules (such as $\mathrm{CO}, \mathrm{H}_{2}$, $\mathrm{O}_{2}, \mathrm{NO}, \mathrm{NH}_{3}$ ) under high vacuumed (UHV) conditions or at elevated pressures and temperatures [5]. In numerous studies, the chemically and physically modified gold layer has been investigated for enhanced adsorption and detection mechanisms, such as stepped/kinked gold surface [7] gold nanorods, gold nanocrystal, CNTs [8] and graphene oxide coupled with gold nanoparticles [9].

Some novel materials such as graphene [10], reduced graphene oxide (rGO) [11], $\mathrm{Au} / \mathrm{Ag} / \mathrm{Au} / \mathrm{chitosan}$-graphene oxide [12], and $\mathrm{MOS}_{2}$ [13] have been explored to enhance the sensitivity and selectivity of SPR sensors. Among them, graphene has emerged as the most promising material owing to its unique properties, such as a high mobility and high surface-to-volume ratio that are beneficial for the efficient adsorption of biomolecules compared to gold. Similarly, the doped graphene oxide (GO) enriched with abundant defect sites on top of a gold layer is also presumed to exhibit an enhanced SPR response [14]. However, the modulation of the optical bandgap and Fermi energy of GO caused by chemical doping result in changes in the optical properties such as refractive index and dielectric constants. These variations could be monitored by SPR sensing techniques.

Recent studies have shown significant improvements in graphene properties by doping it with different heteroatoms such as Florine (F), Oxygen (O) [15], Nitrogen (N) [16], and Boron (B) [17]. The boron-doped graphene oxide has been reported in various applications such as supercapacitor [18], Li-ion batteries [19], solar cells [20], and the enhanced photogenerated catalysis [21]. Moreover, several techniques such as hydrothermal method [22], arc-discharge process [23], and chemical vapor deposition (CVD) [24] have also been employed for the doping of graphene-based materials, where stable atomic substitution with carbon are highly desirable for the tunability of optical and electronic properties of graphene [25]. Among the reduction methods, GO is mostly reduced thermally at high temperatures [26]. However, the reduction at high temperatures produces high-density defects on the edge and basal planes, which deteriorates the electrical and the optical properties [27].

In this work, we have investigated the plausibility of microwave-assisted hydrothermally grown boron-doped reduced graphene oxide (B-rGO) as a promising material to achieve desired properties. The reduction of GO using the microwave-assisted technique is favorable owing to its easy process-ability, short time requirement, high yield, non-requirement of hazardous chemicals, and specialized equipment [27]. The chemical substitutional doping of boron atoms in the $\mathrm{sp}^{2}$ bonded hexagonal structure of the carbon network induces P-type characteristics with an enhanced hole concentration and charged carrier's mobility [28]. The boron doping of graphene also promotes excellent electronic properties by the tunability of the energy bandgap and electrical conductivity [18]. We investigated the optical bandgap tuning capability of B-rGO and its application in surface plasmon resonance. B-rGO was initially synthesized using boric acid via the microwave-assisted hydrothermal technique. The structural and morphological studies of the synthesized B-rGO were then performed using the FESEM, FTIR, XPS, Raman, and XRD techniques. The bandgap study was conducted using UV-Vis spectra and Tauc plot estimations. The SPR responses were investigated experimentally using customize Kretschmann configuration and compared with the corresponding simulated results. The obtained results showed an improvement to the optical bandgap, electrical conductivity, and SPR sensing performance of the B-rGO compared to GO.

\section{Results and Discussion}

\subsection{FESEM Analysis}

Figure 1a,b shows the FESEM image of B-rGO consisting of a planar-like structure. The layered-shape surface of B-rGO contains several wrinkles and corrugations, possibly due to the presence of boron atoms and oxygen-related functional groups. The elemental compositions of 
B-rGO are presented in its dispersive energy X-ray (EDX) spectra, as shown in Figure 1c, indicating the strong signature of carbon $(\mathrm{C})$ atoms. The signatures of boron $(\mathrm{B})$ and oxygen $(\mathrm{O})$ are also visible but not discretely recognized, possibly due to their near-atomic number with $C$ and small concentration. The presence of a few unmarked peaks indicates the presence of platinum (Pt) [29]. The element mapping was also carried out to confirm the element distribution of B-rGO, as shown in Figure $1 \mathrm{~d}-\mathrm{f}$ for elements $\mathrm{C}, \mathrm{O}$, and $\mathrm{B}$, respectively. The homogeneous dispersion of the three identified elements was found to conform in the selected area of $30 \mu \mathrm{m}^{2}$.
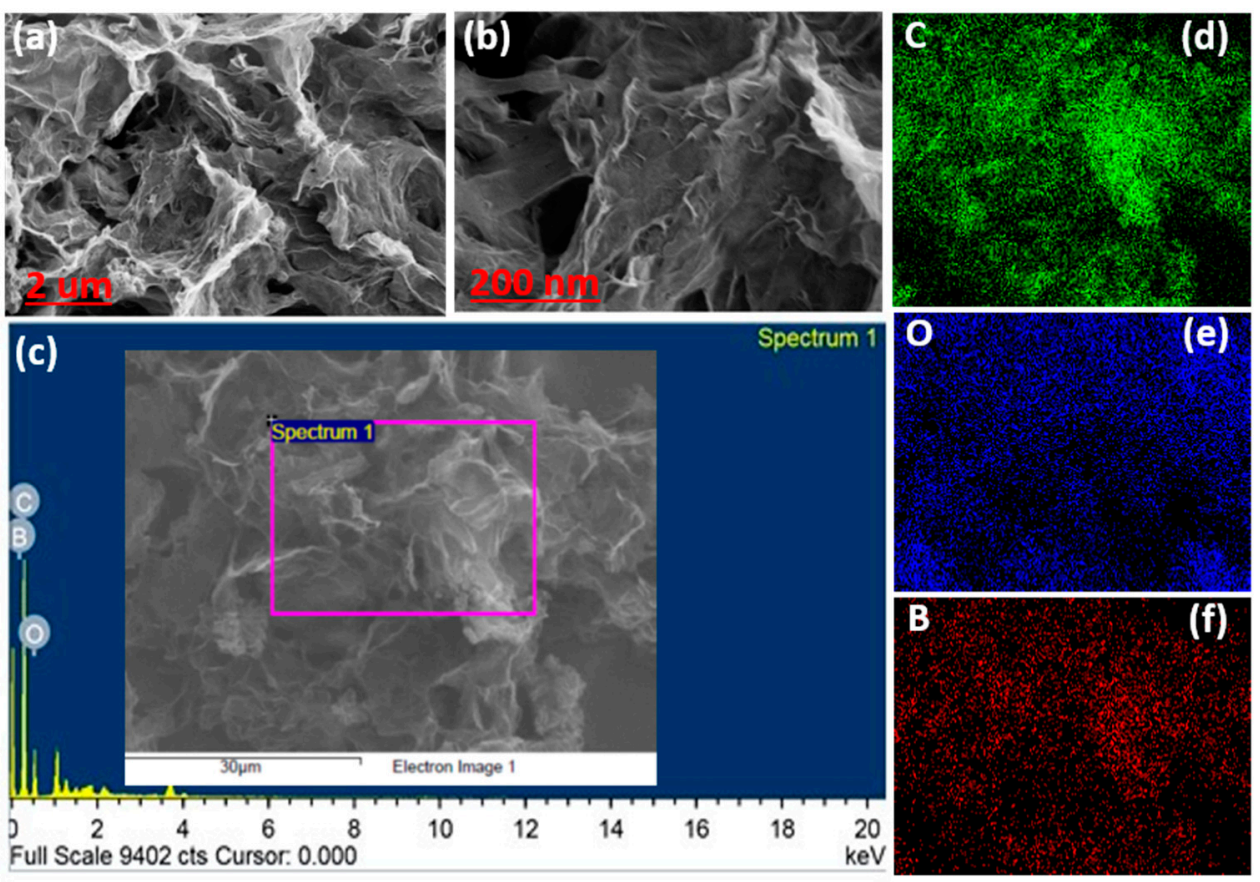

Figure 1. FESEM images of B-rGO at lower (a) and higher (b) magnifications. (c) EDX-spectra of B-rGO. The elemental mapping of (d) carbon, (e) oxygen, and (f) boron.

\subsection{FTIR Spectroscopy}

The synthesis of GO and B-rGO was also tracked using FTIR spectra analysis, as shown in Figure 2a. On account of the GO analysis, a broad FTIR spectrum in range $3700-3000 \mathrm{~cm}^{-1}$ was observed that corresponds to the presence of a hydroxyl group $\left(\mathrm{H}_{2} \mathrm{O}\right.$ and $\mathrm{COOH}$ possibly), $\mathrm{O}-\mathrm{H}$ starching vibrations, and adoption of water molecules [30,31]. The corresponding peaks at 1226, 1418, and $1743 \mathrm{~cm}^{-1} \mathrm{are}$ attributed to the epoxide $(\mathrm{C}-\mathrm{O}-\mathrm{C})$, carboxyl $\left.\mathrm{COOH} / \mathrm{H}_{2} \mathrm{O}\right)$, and ketone $(\mathrm{C}=\mathrm{O})$ groups, respectively $[32,33]$. However, the $(\mathrm{OH})$ bending vibration at $1620 \mathrm{~cm}^{-1}$ contributed due to the absorption of water molecules, which is also attributed to the $(\mathrm{C}=\mathrm{C})$ aromatic vibration [34].

Furthermore, the peak at $1043 \mathrm{~cm}^{-1}$ was assigned to the stretch vibration of $\mathrm{C}-\mathrm{O}$ groups [35]. Subsequently, after microwave-assisted hydrothermal reaction of GO and boric acid, the peaks correlated with the oxygen-containing group were found to be substantially reduced, and even some disappeared. Moreover, new additional peaks were observed in the FTIR spectrum range from 1000 to $1750 \mathrm{~cm}^{-1}$. Therefore, the vibrational band $1735 \mathrm{~cm}^{-1}$ was assigned to the $\mathrm{C}=\mathrm{O}$ functional group, which was slightly shifted to the $1695 \mathrm{~cm}^{-1}$ with lower intensity as compared with the GO spectrum. In the FTIR spectrum of B-rGO, the band around $1040 \mathrm{~cm}^{-1}$ ascribed to the asymmetric B-O-B stretch $\mathrm{B}-\mathrm{O}$ bond among one tetrahedral and one trigonal $\mathrm{B}$ atom [36]. Usually, the B-C functional group was observed from 1050 to $1200 \mathrm{~cm}^{-1}$, where the peak at $1180 \mathrm{~cm}^{-1}$ corresponded to the stretching vibration of the B-C bond. Particularly, the effective downshift in the $C$ network was observed due to the $B$ substitution, which was ascribed to the decreased force constant in the stretching vibration of the B-C bond in comparison with C-C, comparatively [36]. However, the existence of higher C content might have induced the enhanced frequency of B-C stretching [37]. 

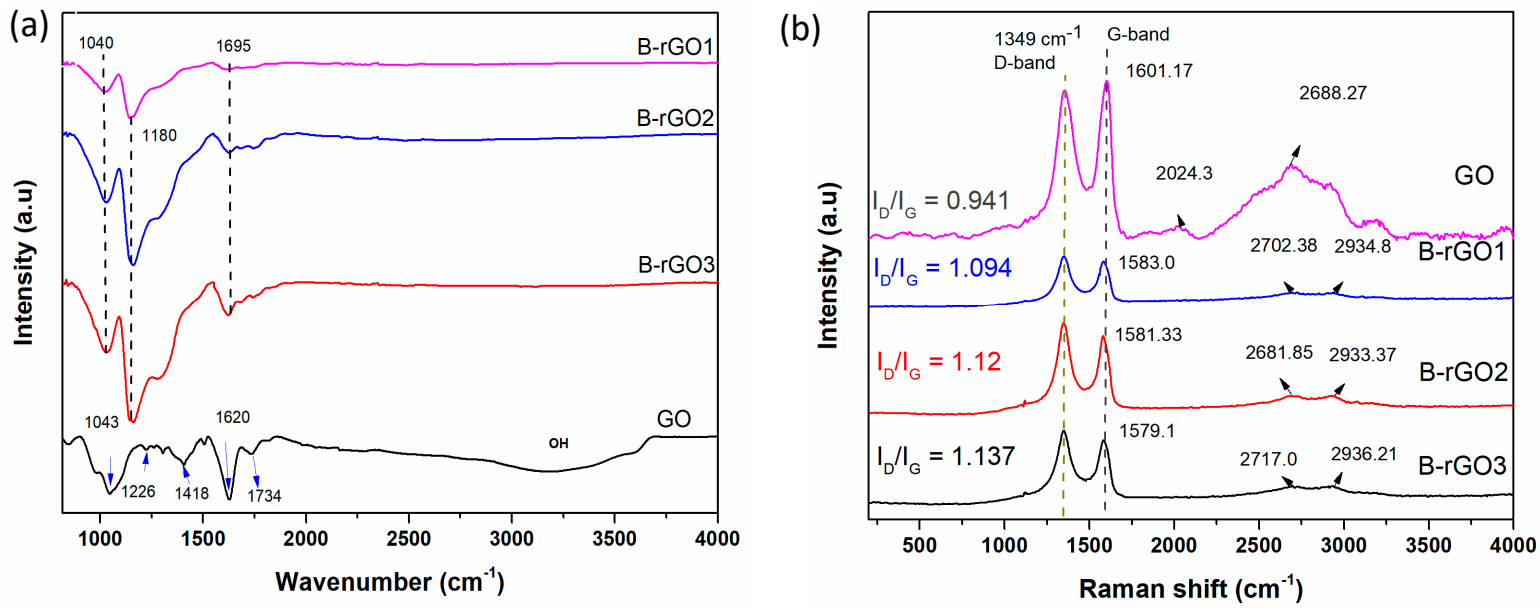

Figure 2. (a) FTIR and (b) Raman spectra of B-rGO1, B-rGO2, B-rGO3, and GO.

\subsection{Raman Spectroscopy}

Figure $2 \mathrm{~b}$ shows the Raman spectra of GO and B-rGO samples at ambient temperatures with $514 \mathrm{~nm}$ laser excitations, where the Raman measurement was carried out from the 0 to the $4000 \mathrm{~cm}^{-1}$ spectrum region. From the Raman spectra of GO, the intensity peaks found at $1349 \mathrm{~cm}^{-1}$ and $1601.17 \mathrm{~cm}^{-1}$ were ascribed to the D- and G- bands, respectively. The D-band at $\sim 1349 \mathrm{~cm}^{-1}$ observed in the GO spectrum, was correlated to the defects and disorders in the graphitic lattice, due to the addition of oxygen-related functional groups. In addition, the second-order D, also known as 2D or $\mathrm{G}^{\prime}$, appeared at $2688 \mathrm{~cm}^{-1}$, attributing to the process of the two second-order phonon vibrations [19]. After the boron doping, a slight redshift in the G-band was observed in B-rGO samples in comparison to GO [38,39]. However, the increment in the D-band intensities in comparison with the G-band intensities of the B-rGO spectra indicated that the extended deformation exhibited the boron doping. Moreover, the intensity ratio $\left(I_{D} / I_{G}\right)$ defined the quality of the material and the relative concentration of $\mathrm{sp}^{3}$ defects in the $\mathrm{sp}^{2}$-hybridized graphene structure [21]. An increment in $\left(I_{D} / I_{G}\right)$ ratio was also observed in the B-doped configurations, i.e., GO (0.94), B-rGO1 (1.094), B-rGO2 (1.12), and B-rGO3 (1.137). The increase in the $I_{D} / I_{G}$ ratio after microwave-assisted hydrothermal treatment indicated the reduction of GO with the existence of boron atoms. The shift of the disorder and graphitic $(G+D)$ peaks at 2934, 2933, and $2936 \mathrm{~cm}^{-1}$, respectively, indicated the existence of few-layer graphene [40]. The shift of the 2D band in the B-rGO spectra also specified the weak (B-C) carbon bonding of $2.57 \mathrm{eV}$ in comparison with the $3.71 \mathrm{eV}$ of $(\mathrm{C}-\mathrm{C})$ bonding [41].

Furthermore, the electrical conductivity measurement was carried out to integrate the Raman information. The electrical conductivity of GO and B-rGO samples was measured at room temperature using Hall-effect measurement (HMS-3000 Series) (see Table S1, Supplementary Material). It was observed that the electrical conductivity of GO increased with the reduction of the oxygen-related functional groups and an increase in $\mathrm{sp}^{2}$ domains. The electrical conductivity of GO was found to be further improved with boron doping, and this might have been dependent on the doping concertation of the incorporated atoms [35]. The increment in the p-type electrical conductivity corresponded to the increase in boron contents in the B-rGO samples. These results were found to be in agreement with previous studies $[27,42]$.

\subsection{XPS Analysis}

The XPS survey scan for GO and B-rGO samples is shown in Figure 3a, which indicates the C1s, O1s, and B1s peaks at 285, 533, and $190 \mathrm{~cm}^{-1}$, respectively. Moreover, reduced O1s peak in the B-rGO survey indicated the reduction of oxygen-related groups in the GO-modified structure. Figure 3c shows the Gaussian deconvolution of the O1s spectrum that could be split into four peaks 531, 532, 533, and $534 \mathrm{eV}$, confirming to the $\mathrm{O}=\mathrm{C}-\mathrm{OH}, \mathrm{C}-\mathrm{OH}$, and $\mathrm{C}-\mathrm{O}$, functional groups, respectively [43]. 
Moreover, the atomic percentage of each element was also determined from the XPS spectrum analysis of GO and each of the three B-rGO samples, as summarized in Table 1. In these studies, the atomic percentage of boron contents in the B-rGO samples was found to be 4.80, 5.53, and 6.51 at.\%, respectively, showing an agreement along with the previously reported work [19,44,45]. The maximum boron doping concentration, up to 6.51 at.\% was confirmed through XPS analysis, where B-rGO samples with lower doping could also be synthesized by varying the concentration of boric acid and GO in the synthesis process. More interestingly, a decrement in the atomic percentage of oxygen was observed, where the atomic percentage of oxygen for the GO, B-rGO1, B-rGO3, and B-rGO3 samples was determined to be $34.15,11.46,10.27$, and 9.58 at. \%, respectively. Table 2 presents each functional group determined from the deconvolution of the C1s, O1s, and B1s XPS peaks, respectively.

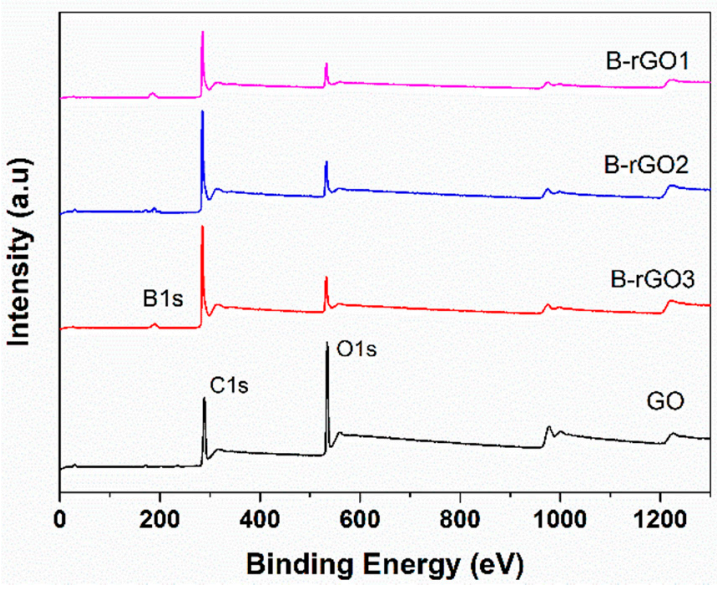

(a)

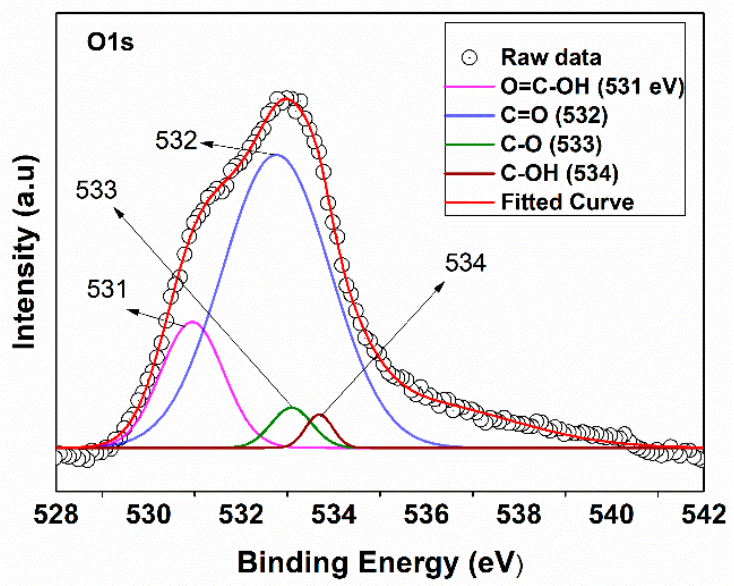

(c)

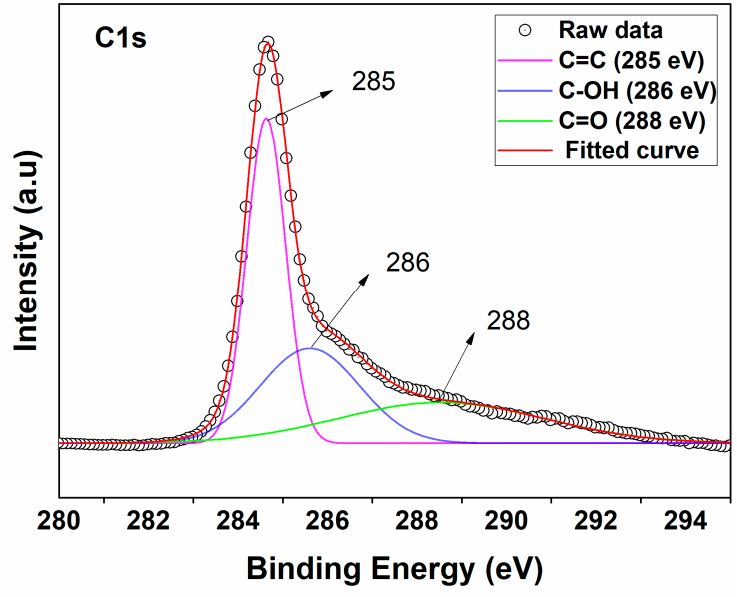

(b)

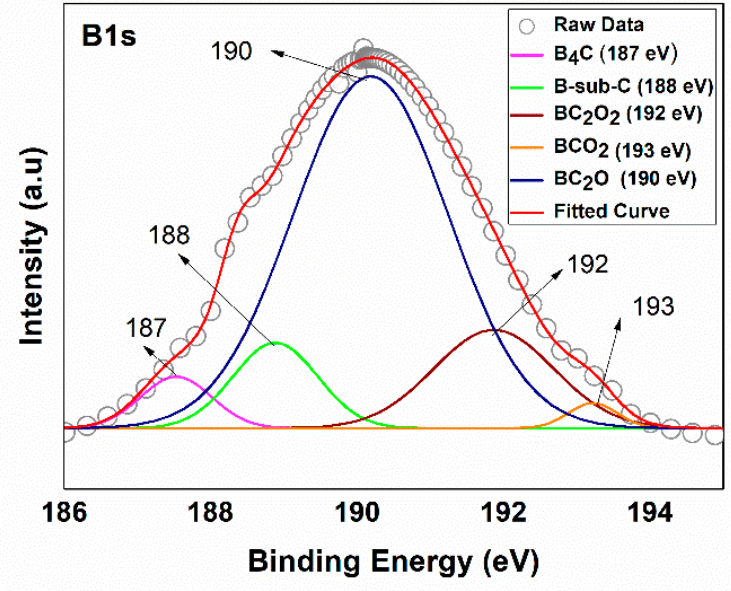

(d)

Figure 3. (a) XPS survey analysis of GO and B-rGO samples (B-rGO1, B-rGO2, and B-rGO3, respectively). From (b) to (c) depicts the XPS deconvoluted peaks of C1s (b), O1s (c), and B1s (d) observed from XPS analysis of B-rGO.

Table 1. The at.\% of each element in each B-rGO samples determined in the XPS analysis.

\begin{tabular}{cccc}
\hline Material & C (at.\%) & O (at.\%) & B (at.\%) \\
\hline GO & 65.78 & 34.15 & - \\
B-rGO1 & 83.67 & 11.46 & 4.80 \\
B-rGO2 & 84.16 & 10.27 & 5.53 \\
B-rGO3 & 83.85 & 9.58 & 6.51 \\
\hline
\end{tabular}


Table 2. The XPS peak assigned to each functional group.

\begin{tabular}{ccc}
\hline Peak's & Peak (eV) & Assignment \\
\hline \multirow{2}{*}{ C1s } & 285 & $\mathrm{C}=\mathrm{C}$ \\
& 286 & $\mathrm{C}-\mathrm{OH}$ \\
288 & $\mathrm{C}=\mathrm{O}$ \\
& 531 & $\mathrm{O}=\mathrm{C}-\mathrm{OH}$ \\
O1s & 532 & $\mathrm{C}=\mathrm{O}$ \\
& 533 & $\mathrm{C}-0$ \\
& 534 & $\mathrm{C}-\mathrm{OH}$ \\
& 187 & $\mathrm{~B} 4 \mathrm{C}$ \\
$\mathrm{B} 1 \mathrm{~s}$ & 188 & $\mathrm{~B}-\mathrm{Sub}-\mathrm{C}$ \\
& 190 & $\mathrm{BC} 2 \mathrm{O}$ \\
& 192 & $\mathrm{BC} 2 \mathrm{O} 2$ \\
& 193 & $\mathrm{BCO} 2$ \\
\hline
\end{tabular}

The deconvolution of the $\mathrm{C} 1$ s spectra in the $\mathrm{B}-\mathrm{rGO}$ indicated several peaks comprising of $\mathrm{C}=\mathrm{C}$ $(285 \mathrm{eV}), \mathrm{C}-\mathrm{OH}(286 \mathrm{eV})$, and $\mathrm{C}=\mathrm{O}(288 \mathrm{eV})$, related to the various chemical bonding carbon in the reduced graphene oxide structure. Especially, an intense peak in the $\mathrm{C} 1 \mathrm{~s}$ spectra at $284.75 \mathrm{eV}$ corresponded to the $\mathrm{C}-\mathrm{C} \mathrm{sp}{ }^{2}$ bonded graphite carbon structure. It was also implied that the majority of carbon atoms were in honeycomb structure [19]. Figure 3d illustrates the XPS spectrum B1s of B-rGO. The Gaussian deconvolution of the entire B1s spectrum showed that the entire spectra could be deconvoluted in five peaks sequentially. The B1s peak at 191 eV was found to be higher than the pure boron peak of $188 \mathrm{eV} \mathrm{[46],} \mathrm{indicating} \mathrm{the} \mathrm{existence} \mathrm{of} \mathrm{boron} \mathrm{in} \mathrm{the} \mathrm{oxidation} \mathrm{states.} \mathrm{The} \mathrm{deconvoluted}$ peaks of the $\mathrm{B} 1 \mathrm{~s}$ also indicated the $\mathrm{B}_{4} \mathrm{C}(187 \mathrm{eV}), \mathrm{BC}_{2} \mathrm{O}_{2}(188 \mathrm{eV}), \mathrm{BC}_{2} \mathrm{O}(190 \mathrm{eV})$, and $\mathrm{BCO}_{2}(193 \mathrm{eV})$ peaks, confirming the successful doping of the $\mathrm{GO}$ carbon network with boron atoms [35]. The B-sub-C $(188 \mathrm{eV})$ deconvoluted peak referred to the substitution doping of $\mathrm{B}$ atoms with $\mathrm{C}$ atoms in the graphite structure [47]. The XPS study of B-rGO revealed that GO was not fully reduced, and the synthesized B-rGO samples still contained some oxygen-related functional groups.

\subsection{XRD Analysis}

The crystalline structure of $\mathrm{GO}$ and boron $\mathrm{B}-\mathrm{rGO}$ has been investigated by the XRD diffraction pattern. Figure 4 shows the XRD pattern of the synthesized GO, doped B-rGO1, B-rGO2, and B-rGO3 powder samples. The (001) strong and diffraction peak for GO was observed at $11^{\circ}=2 \theta$ [48]. After boron doping, the (001) peaks had disappeared, and the XRD diffraction peaks (002) for B-rGO1, B-rGO2, and B-rGO3 appeared at $25.93^{\circ}, 25.95^{\circ}$, and $26^{\circ}=2 \theta$, respectively, showing an agreement with previously reported data [49]. Also, the lower XRD peaks (001) were also observed for all B-rGO samples at $43^{\circ}$.

Moreover, the interlayer distance between the wave field and the crystalline plane was determined by using Bragg's Equation (1) [50],

$$
\lambda=\frac{2 d \sin (\theta)}{n}
$$

where, $\theta, \lambda, n$, and $d$ are the scattering angle, the wavelength of the incident $\mathrm{X}$-ray $(1.54 \AA)$, the order of reflection, and the interlayer distance, respectively. The interlayer distance of GO $\sim 8.05 \AA$ showed an agreement with reported work $[51,52]$. Higher layer distance in the synthesized GO was observed due to the repulsive forces induced by the presence of oxygen-related functional groups and the water molecules. Moreover, the interlayer distance of $4.21 \AA, 4.18 \AA$, and $4.15 \AA$ were determined for the B-rGO1, B-rGO3, and B-rGO3, respectively. The decrement in the interlayer distance, as observed in the XRD spectra of B-rGO, was attributed to the increment in boron concentration and also resulted from the decrement in a considerable amount of oxygen-related functional groups [35]. 


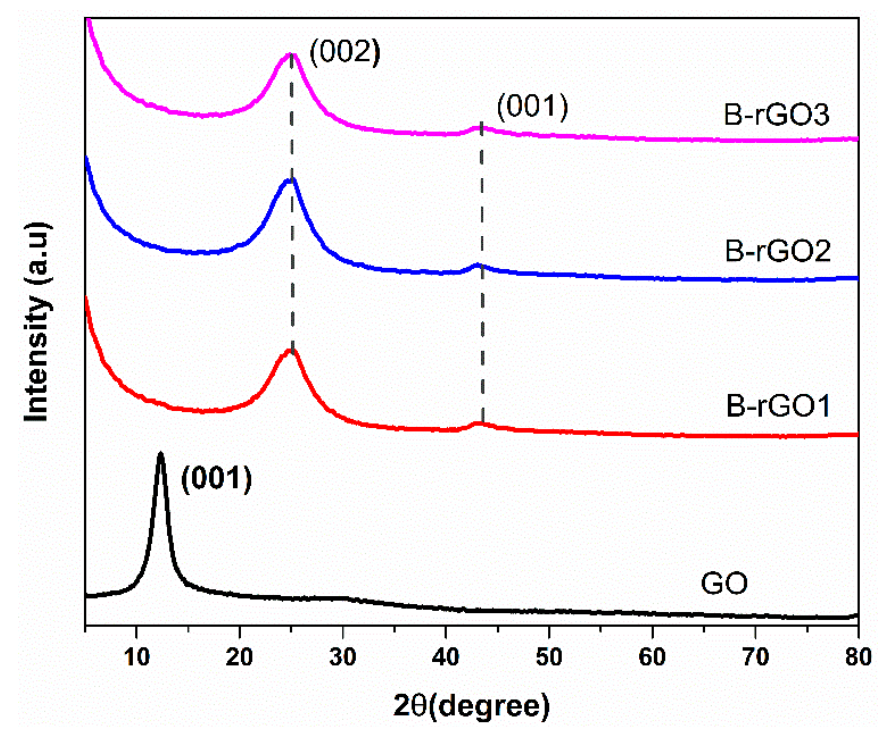

Figure 4. The XRD pattern of the synthesized GO, doped B-rGO1, B-rGO2, and B-rGO3 powder samples.

\subsection{Optical Bandgap Studies}

The optical bandgap of B-rGO samples was extracted using ultraviolet-visible (UV-Vis) absorption spectral analysis and the Tauc plots, as shown in Figure 5. UV-Vis spectra of all B-rGO samples (Figure 5a) display the absorption peaks from 375, 382, and $386 \mathrm{~nm}$ due to $\pi$ to $\pi^{*}$ transitions, which corresponds to the direct optical bandgap 2.91 to $3.05 \mathrm{eV}$ [53]. The lower light absorption was observed from 450 to $800 \mathrm{~nm}$, attributing to the maximization of $\mathrm{sp}^{2}$ domains in the B-rGO. Moreover, the extra shoulder in the UV-Vis spectra observed from $375 \mathrm{~nm}$ to $386 \mathrm{~nm}$ for B-rGO samples correlated to the $\mathrm{n}$ to $\pi^{*}$ transitions $\mathrm{C}=\mathrm{O}$ bonds [54]. This corresponded to the electrons transition from boron states to the $\pi^{*}$ states, indicating the heterogeneous distribution of boron atoms in the carbon network of reduced graphene oxide. It was also interesting to note that the UV-VIS absorption spectra of the B-rGO samples showed strong absorption of light in the region of the UV spectrum towards almost visible.

The optical bandgap sketched for GO, B-rGO1, B-rGO2, and B-rGO3 is shown in Figure $5 \mathrm{~b}$ to $5 \mathrm{e}$, where the value of the direct energy bandgap was inferred by extrapolating the linear part of the UV-Vis absorption curve to zero x-axes. From the Tauc plot calculation based on Equation (6), the direct bandgap values for the GO, B-rGO1, B-rGO2, B-rGO3 samples were determined to be $\sim 3.41,3.05,2.94$, and $2.91 \mathrm{eV}$, respectively. It was also determined that the B-rGO3 samples with a maximum boron doping concentration of 6.51 at. \% depicted the lowest optical bandgap value $(2.91 \mathrm{eV})$, comparatively. Moreover, the direct optical bandgap was further investigated with the increment of boron doping concentration, where the bandgap was found to be reduced, attributing to the reduction of the oxygen-related functional groups in the rGO carbon network. The observed bandgap reduction could also be ascribed to the compensation of energy states associated with the incorporation of boron dopant atoms, that ultimately shift the direction of the conduction band edges [55]. 

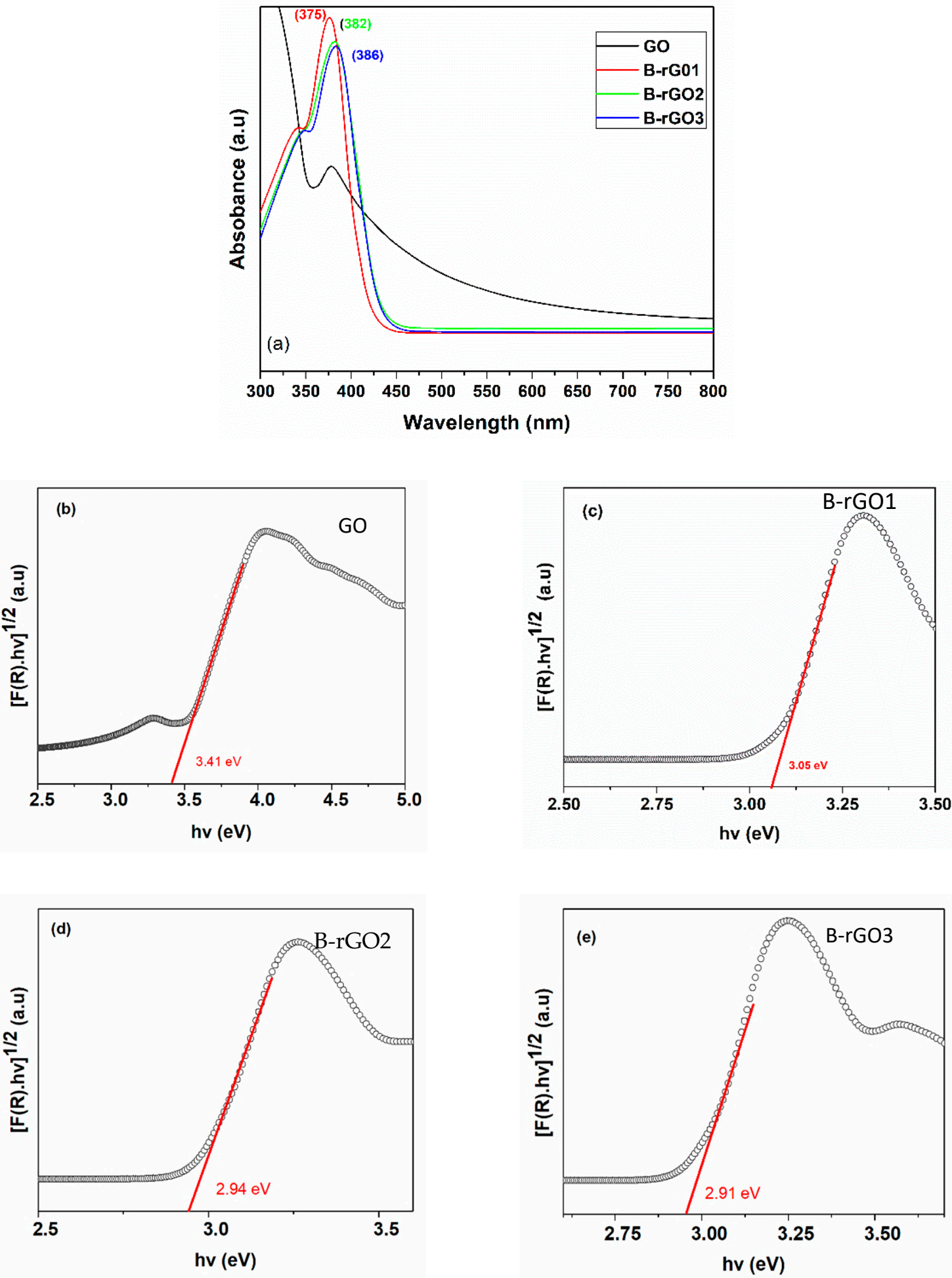

Figure 5. (a) UV-Vis absorption spectra of GO and B-rGO samples (b)-(e) Tauc plot for the GO, and B-rGO1, B-rGO2, and B-rGO3 samples, respectively.

\subsection{Surface Plasmons Resonance (SPR) Study of B-rGO}

The optical constant for the boron-doped reduced graphene thin film samples (B-rGO1, B-rGO2, and B-rGO3), particularly the real part of the dielectric constant and complex refractive index was determined by applying Equations (2)-(11) at $633 \mathrm{~nm}$, as shown in Table 3. Where the $633 \mathrm{~nm}$ laser source was conventionally used for gold-based SPR sensing applications [56]. Besides, the values 
of the optical constants of the gold and the glass prism (SF11) are also included in Table 3 for the SPR studies. Prior to the SPR study of B-rGO, the thickness of the Au layer was optimized for the minimum reflectance $R_{m}$, as presented in Figure 6a. The optimization of $R_{m}$ was performed to ensure the maximum surface plasmon excitation. The optimal SPR response with the deepest curve was determined from the gold thin film with 50 nm thickness, as shown in Figure 6a, while the same thickness value was considered for the later studies.

The constant optical values of the B-rGO samples were decreased in comparison to that of GO and rGO, as shown in Table 3, attributing to the possible reduction of oxygen-related functional groups in the B-rGO after doping. On the other hand, the depth and width of the SPR reflectivity curve were affected by the change in the values of the optical constants and the thickness (d) of the light-absorbing surface (gold surface), as shown in Figure 6b [59]. It could be observed that the SPR curves for B-rGO1, B-rGO2, and B-rGO3 were overlapped due to the marginal difference in their constant optical values. To assess the SPR sensing performance of GO and B-rGO samples, the parameter FWHM, and the value of the penetration depth through the dielectric medium $\delta_{d}$ were investigated. Particularly, the high-performance SPR sensing layer was expected to possess low FWHM and high $\delta_{d}$ values [2]. Figure $6 \mathrm{c}$ shows the variation of the FWHM for the gold, GO, thermally reduced GO, B-rGO1, B-rGO2, and B-rGO3-based SPR sensors, attained using Equation (18). GO showed the lowest FWHM response as compared with B-rGO samples (see Table 3 ). In Figure $6 \mathrm{~d}$, the $\delta_{d}$ relative values calculated from Equation (19) for the gold, GO, thermally reduced GO, B-rGO1, B-rGO2, and B-rGO3 samples were found to be $359.64 \mathrm{~nm}, 103.82 \mathrm{~nm}, 100.57 \mathrm{~nm}, 128.85 \mathrm{~nm}, 128.16 \mathrm{~nm}$, and $127.63 \mathrm{~nm}$, respectively.

The measured thickness of the Au and B-rGO layers was found to be $50 \pm 1.5 \mathrm{~nm}$ and $5 \pm 1 \mathrm{~nm}$, respectively. In Figure 6e, the experimental and simulated SPR responses of the B-rGO1, B-rGO2, and B-rGO3 were compared. The position of the SPR angle for the measured and simulated B-rGO1, B-rGO2, and B-rGO3-based SPR sensors were found to be almost the same. However, the SPR curves for the simulated sensors were deeper as compared to the measured values. This could be attributed to the slight variation in the thickness of the $\mathrm{Au} / \mathrm{B}$-rGO layer and the refractive index values of the SF11 Prism and index matching liquid. Interestingly, the B-rGO demonstrated the highest values of $\delta \mathrm{d}$, compared to the GO, and the thermally reduced rGO. This shows that B-rGO-based sensors could produce an enhanced SPR response.

Table 3. The optical constants values for the GO, rGO, and B-rGO samples at $633 \mathrm{~nm}$.

\begin{tabular}{ccccc}
\hline Material & Refractive Index $\boldsymbol{n}$ & Extinction Coefficient $\boldsymbol{k}$ & Dielectric Constant & Reference \\
\hline Prism (SF11) & 1.7786 & 1.0026 & - & {$[13]$} \\
Gold Au & 0.183 & 3.433 & - & {$[57]$} \\
Graphene oxide & 1.957 & 0.0101 & - & {$[58]$} \\
(GO) & & & - & {$[58]$} \\
Thermally reduced & 1.993 & 0.243 & 3.056 & Equations (2)-(11) \\
GO (rGO) & 1.733 & 0.231 & 3.079 & Equations (2)-(11) \\
B-rGO1 & 1.738 & 0.243 & 3.100 & Equations (2)-(11) \\
B-rGO2 & 1.742 & 0.257 & & \\
B-rGO3 & & &
\end{tabular}



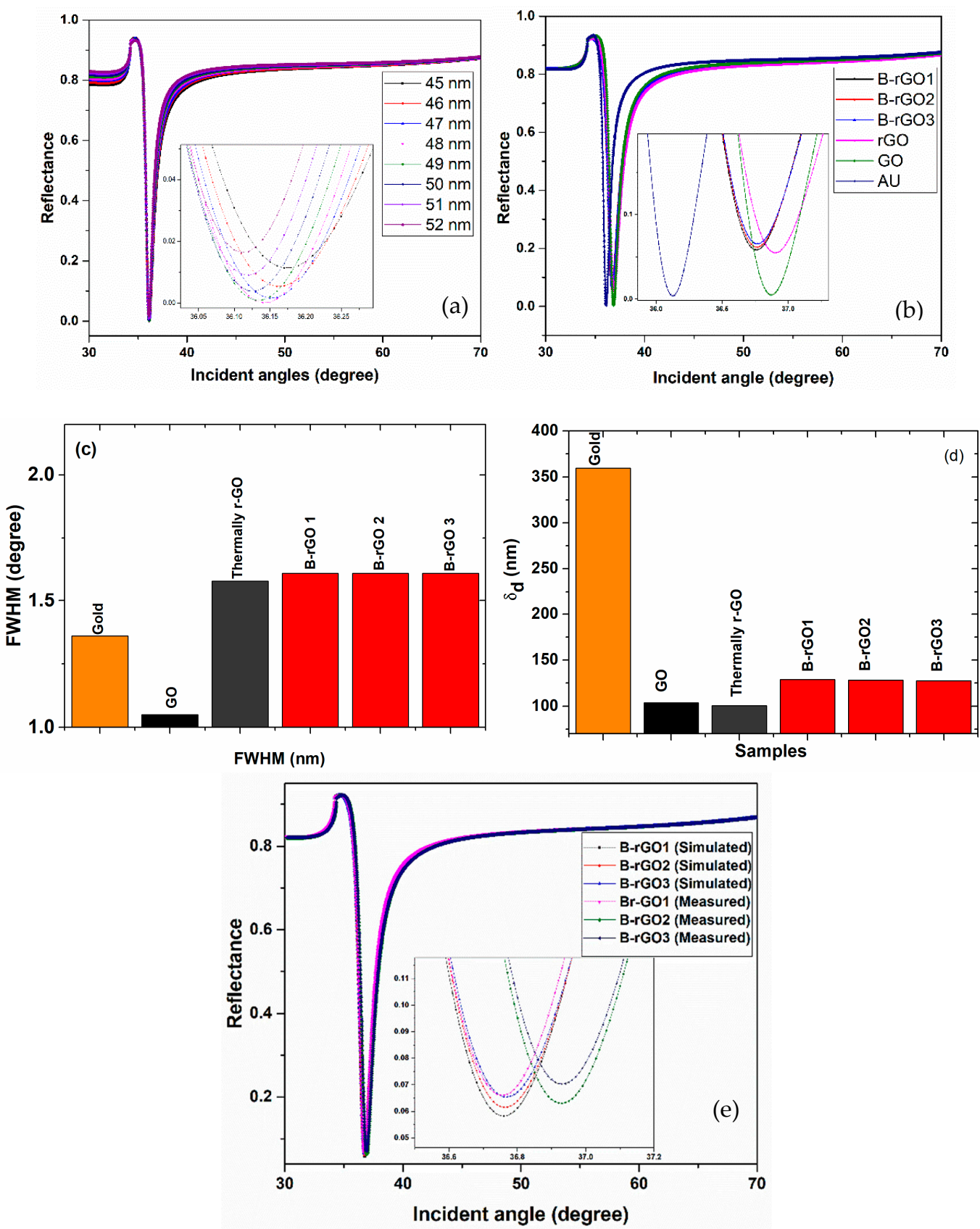

Figure 6. (a) SPR curves for the optimization of gold film thickness. (b) SPR curves for gold, GO, thermally reduced GO, B-rGO1, B-rGO2, and B-rGO3-based SPR sensors. (c) Variations of FWHM for gold, GO, thermally reduced GO, B-rGO1, B-rGO2, and B-rGO3 based SPR sensors (d) Variations of dielectric penetration depth in for gold, GO, thermally reduced GO, B-rGO1, B-rGO2 and B-rGO3-based SPR sensors. (e) Comparative study of the simulated and measured SPR response for B-rGO1, B-rGO2, and B-rGO3-based sensors.

\section{Materials and Methods}

\subsection{Materials}

Graphite flakes $(\sim 50 \mu \mathrm{m})$, ethanol $(95.0 \%)$, hydrochloric acid $(\mathrm{HCl})(37 \%)$, sulfuric acid $\left(\mathrm{H}_{2} \mathrm{SO}_{4}\right)$ (97\%), phosphoric acid $\left(\mathrm{H}_{3} \mathrm{PO}_{4}\right)(85 \%)$, potassium permanganate $\left(\mathrm{KMnO}_{4}\right)(99.5 \%)$, hydrogen peroxide $\left(\mathrm{H}_{2} \mathrm{O}_{2}\right)(30 \%)$, and other analytical grade reagents purchased from R\&M, Selangor, Malaysia. Boric acid (ACS reagent, 99.5\%, Darmstadt, Germany) was purchased from Sigma-Aldrich. Graphene oxide (GO) 
was synthesized by improved Hummer's method [60], and the boron-doped-reduced graphene oxide was produced by the facile microwave-assisted hydrothermal method using boric acid.

\subsection{Synthesis of Graphene Oxide}

Initially, $360 \mathrm{~mL} \mathrm{H}_{2} \mathrm{SO}_{4}$ and $10 \mathrm{~mL} \mathrm{H}_{3} \mathrm{PO}_{4}$ were mixed and stirred for $2 \mathrm{~h}$ in a $1000 \mathrm{~mL}$ round bottom flask. Three grams of graphite flakes and $18 \mathrm{~g}$ potassium permanganate were then added to the mixed solution $\left(\mathrm{H}_{2} \mathrm{SO}_{4}+10 \mathrm{~mL} \mathrm{H}_{3} \mathrm{PO}_{4}\right)$, which formed a dark green solution. The process was carried out in an ice bath to prevent overheating and explosion. The existing solution was stirred for $12 \mathrm{~h}$ to oxidize and exfoliate the graphite into a few layers of GO structure. After the completion of the reaction, $\mathrm{H}_{2} \mathrm{O}_{2}$ was added to stop the further reactions. At that point, the solution color was changed into a dark yellow-brown. Furthermore, $1 \mathrm{~L}$ of deionized (DI) water was used to dilute the solution, and then its $\mathrm{pH}$ level was adjusted $\sim 1$. The obtained mixture was then centrifuged at $5000 \mathrm{rpm}$ to collect the graphite oxide and decant away the acid. The obtained oxidized graphite flake was further washed with the $10 \% \mathrm{HCl}$ to remove the impurities like potassium and manganese ions, which are typically trapped in the solid. Afterward, the remaining mixture solution was washed numerous times with DI water while waiting for the $\mathrm{pH}$ level to reach $\sim 1$. GO flakes were then collected by a centrifugation process at 10,000 rpm. The GO flakes were dried in a Freeze Dryer and ground into a fine powder. At this stage, the powder was exfoliated into a few and single layers, so it was referred to as graphene oxide $(\mathrm{GO})$ powder.

\subsection{Synthesis of $B-r G O$}

The GO solution $(70 \mathrm{mg} / 100 \mathrm{~mL})$ was prepared in DI water followed by continuous stirring for $2 \mathrm{~h}$ and sonication for $0.5 \mathrm{~h}$ (Scheme 1). One to $3 \mathrm{~g}$ of boric acid solution was also prepared in $10 \mathrm{~mL}$ ethanol by magnetic stirring at $60^{\circ} \mathrm{C}$. After uniform dispersion of boric acid in ethanol, both obtained solutions were mixed and stirred for $8 \mathrm{~h}$ at $80^{\circ} \mathrm{C}$. Subsequently, the obtained mixture solution was poured into $200 \mathrm{~mL}$ Teflon tube autoclave and heat-treated in the oven at $180^{\circ} \mathrm{C}$ for $12 \mathrm{~h}$. The obtained B-rGO flakes were further exposed to microwave energy radiation using the $800 \mathrm{~W}$ microwave oven for the $40 \mathrm{~s}$. The intensity of the microwave intensity was adjusted to an optimized level to enhance the further reduction and absorption of unreacted boron contents. The resulting B-rGO powder was washed several times with DI water to remove untreated boric acid contents. Finally, the dry B-rGO powder was obtained by the dry freezing process in a vacuum oven at $60^{\circ} \mathrm{C}$ for $36 \mathrm{~h}$. The three samples of boron-doped reduced graphene (B-rGO1, B-rGO2, and B-rGO3) were synthesized with three different concentrations of boric acid, 1, 2, and $3 \mathrm{~g}$, respectively.

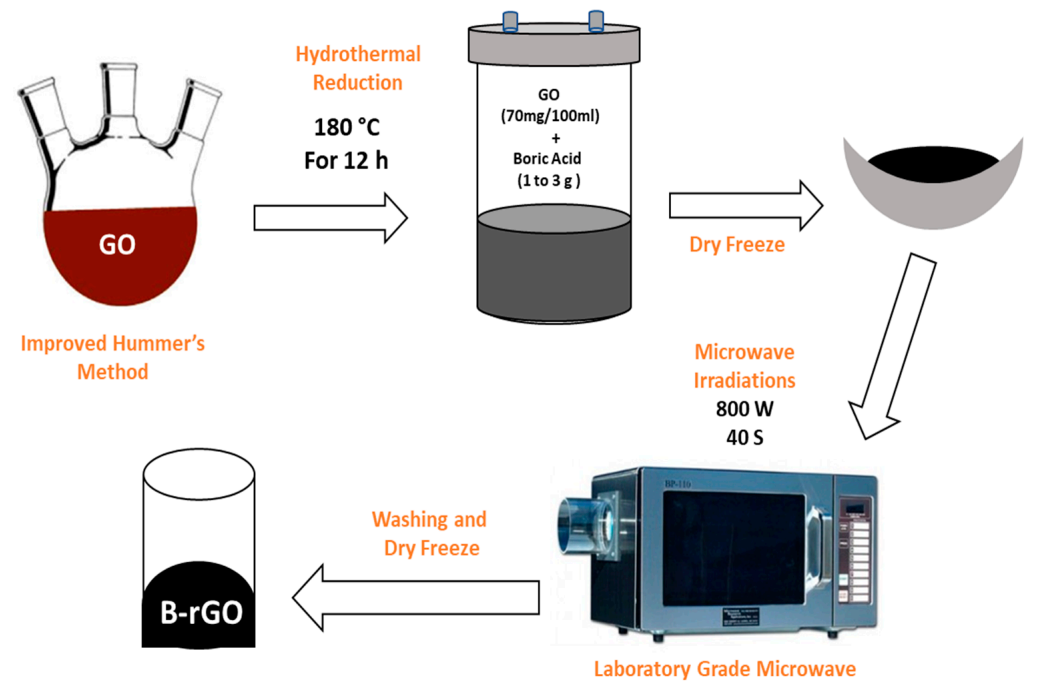

Scheme 1. Microwave Assisted Hydrothermal Approach for Synthesis of Boron-Doped-reduced Graphene Oxide. 


\subsection{Material Characterizations}

Morphology of the B-rGO, with energy dispersive X-ray (EDX) component mapping, was examined by field emission scanning electron microscopy (FESEM, Zeiss Supra 55 VP, Cambridge, UK). The functionalized surface of the GO and B-rGO thin film was studied by Fourier transform infrared (FTIR) spectroscopy (Bruker Instruments, model Aquinox 55, Ettlingen, Germany). Raman spectroscopy (Bruker Instruments, Horiba Jobin Yvon HR800, Ettlingen, Germany) was also performed to reconfirm the arrangement of the composites. The imperfections and defects in the GO and B-rGO samples were also analyzed using Raman spectroscopy. In Raman spectra of GO and B-rGO, various peaks with different intensities appeared, where each peak to the material, followed the imprints and grouping of deformities in materials.

The optical characterization of spin-coated GO and B-rGO thin film was performed by UV-Vis Spectrophotometer (Cary 100, Agilent Technologies, Santa Clara, CA, USA) at room temperature. The thickness estimation was obtained from directly utilizing the surface harshness analyzer (SV-Mitutoyo-3000, Aurora, IL, USA). The XRD estimation was also conducted for additional affirmation of crystallinity and synthesis of GO and B-rGO powder samples by utilizing XRD (X'Pert3 Powder, Westborough, MA, USA). The XRD analysis was performed over a $5-90^{\circ}$ range with step size $0.01 / \mathrm{s}$. Likewise, the existence of various functional groups was also confirmed through the support of X-ray photoelectron spectroscopy analysis (XPS; Thermo logical, K-alpha, Waltham, MA USA). The XPS survey spectra were recorded from 0 to $1200 \mathrm{eV}$, under pressure conditions of $10^{-8}$ mbar through monochromatic $\mathrm{Al}$ Ka radiation $(1486.6 \mathrm{eV})$. The XPS spectra were further analyzed with the help of the Origin (version 9.0, Waltham, MA, USA) software. The deconvolution of multiple peaks was performed on XPS raw data. The existence of individual functional groups in XPS spectra was also analyzed from multiple peaks by the implication of Gaussian curve fittings, where the estimation of the baseline was performed by utilizing the adjacent-averaging technique. The electrical conductivity was measured at room temperature using a Hall Effect measurement (HMS-3000 series, Phoenix, AZ, USA).

\subsection{Optical Bandgap and Optical Constant Measurement}

The reduced optical bandgap energy of B-rGO indicates the prospective utilization of the material in optoelectronic device applications. The optical bandgap and optical constants of the B-rGO samples were obtained from the UV-Vis spectrum of a thin film. The GO and B-rGO dispersion solutions in N-Methyl-2-pyrrolidone (NMP) with a concentration of $0.25 \mathrm{mg} / \mathrm{mL}$ were used for the thin film deposition. The process was complemented by sonication or $0.5 \mathrm{~h}$ followed by the strong magnetic stirring for $2 \mathrm{~h}$. Before spin coating, the obtained dispersed solution was centrifuged at $6000 \mathrm{rpm}$ to segregate the undissolved B-rGO particles. Later, B-rGO based thin film was prepared by using a spin coater (POLOSTM) at 3,000 rpm. From a theoretical perspective, the relationship among the parametric values obtained from UV-Vis spectroscopy, namely; absorption (A), transmission (T), and reflectance (R) could be defined by Equation (2) [61].

$$
R+T+A=1(A=1-R-T)
$$

The Bear-Lambert law (Equation (3)) establishes the relationship between light attenuation through the specific material and its properties, where the relation between transmittance and absorbance of light could be defined [62]. The transmittance and absorption coefficient could be determined by using Equations (4) and (5), accordingly [63].

$$
\begin{gathered}
I(d)=I_{o} e^{-a d} \\
T=(1-R)^{2} e^{-a d} \\
a=\frac{2.303 A}{d}
\end{gathered}
$$


where $I(d)$ is the intensity at a depth of thickness $d, I_{0}$ is the intensity at zero thickness, and $a$ is the absorption coefficient. The optical bandgap structure and the type of electronic transitions could be obtained from the absorption coefficient [64]. The optical bandgap $\left(E_{g}\right)$ could be determined by using Equation (6).

$$
E_{g}=h v-\left(\frac{a h v}{\beta}\right)^{\frac{i}{j}}
$$

where $h v$ is the photon energy, $E_{g}$ is the energy bandgap; $a$ is the absorption coefficient, and $\beta$ is a constant for disorder parameter which is independent of photon energy. The value of parameter $j$ denotes the nature of transition, where the parametric value of $J, 1 / 2$ used for direct allowed transitions, and 3/2 used for the direct forbidden transitions. However, the $j$ parametric value of 2 is described for indirect allowed transitions, and 3 defines the indirect forbidden bandgap transitions [65]. The boron-doped graphene possesses direct electronic transitions [66]. Therefore, in this study, the optical bandgap was determined from the Tauc plot estimation, i.e., the optical bandgap $(h v)$ verses $(a h v)^{2}$. Furthermore, the imaginary part of the refractive, $K$ (extinction coefficient) correlated with an absorption coefficient by the Equation (7).

$$
k=\frac{a \kappa}{4 \pi}
$$

where $\lambda$ is the wavelength of the light wave. Based on the UV-Vis reflectivity measurement, the real part of the refractive index $(n)$ could also be obtained from the Fresnel Equation (8) based on the reflectivity measurement using Equation (9).

$$
\begin{gathered}
R=\frac{(n-1)^{2}+k^{2}}{(n+1)^{2}+k^{2}} \\
n=\left[\frac{1+R}{1-R}\right]+\sqrt{\frac{4 R}{(1-R)}}-k^{2}
\end{gathered}
$$

The value of the complex dielectric of B-rGO could also be evaluated from the value of $K$, and $n$.

$$
\begin{gathered}
\varepsilon_{r}=n^{2}+k^{2} \\
\varepsilon_{i}=2 n k
\end{gathered}
$$

where $\varepsilon_{r}$ and $\varepsilon_{i}$ are the real and imaginary parts of the B-rGO dielectric constant, respectively.

\subsection{Surface Plasmons Resonance (SPR) Study}

The surface plasmons resonance of the prepared samples was studied using our customized system, which was entirely based on the Kretschmann configuration, as shown in Figure 7a. The polarizer is an optical filter that is utilized to P-polarize the incident light beam in the direction parallel to the plane of incidence. However, in this case, the direction was within the horizontal plane. The modified Kretschmann configuration was used to excite the surface plasmon in which metallic film was in direction with a prism base. The chopper (SR 540) and lock-in amplifier (SR 530) was incorporated within the proposed system to optimize the measuring result in terms of signal to noise ratio (SNR). Besides, in this configuration, the chopper was used for the intensity modulation of the incident light, and the lock-in amplifier was used to measure the corresponding data to the chopping frequency. To evaluate the change over the momentum in incident light regarding the dielectric-metal interface, the prism $(n=1.77861)$ was fixed on the rotation table and circled with a step size of $0.1^{\circ}$ using a stepper motor (Newport MM 3000, Scotia, NY, USA). In this system, the He-Ne (633 nm, $5 \mathrm{~mW})$ laser beam was utilized with a photodiode detector. 


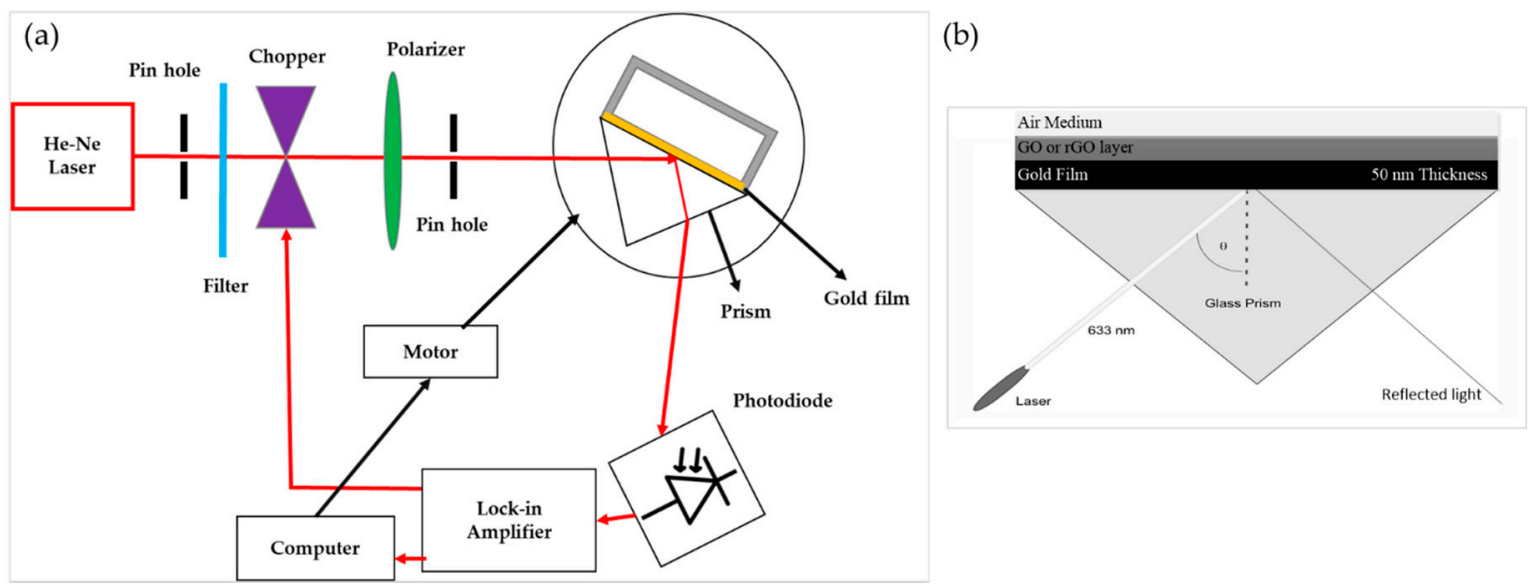

Figure 7. (a) The schematic diagram of the customized Kretschmann configuration-based system for SPR measurements, (b) the SPR-based sensing system.

The existence of resonating free electrons with the collective oscillation on the plasma surface (i.e., metal surface) may induce charge density transverse waves propagation along the plasma surface. The oscillation of the transverse wave normal to the plasma surface is called surface plasmons. The surface plasmons resonance gets excited when the electromagnetic waves are coupled through the particular medium on the metallic surface. The light source must be P-polarized to the plasma surface, where the SPs only have the electric field component normal to the surface [67]. Consequently, the SPs get excited when the momentum between the surface plasmons (SPs) and the incident light is matched. The maximum energy transfer between SPs and photons occurs at the SPR angle, which mainly depends on the dielectric property of adjacent material on the gold thin film surface, and it is stated by $K_{s p}$ (surface plasmons vector) as shown in Equation (12).

$$
K_{s p}=\frac{\omega}{c} \sqrt{\frac{\varepsilon_{1} \varepsilon_{2}}{\varepsilon_{1}+\varepsilon_{2}}}
$$

where $c$ is the velocity of light, $\omega$ is the frequency and $\varepsilon_{m}, \varepsilon_{d}$ are the dielectric constant for the metal and dielectric material, respectively. The electric field is perpendicular to the Au-coated thin film. The relationship between the dielectric constant and refractive index could be defined by Equation (13).

$$
\varepsilon=n^{2}
$$

Equation (12) can be rewritten as,

$$
K_{s p}=\frac{2 \pi}{\lambda} \sqrt{\frac{n_{2}^{2} n_{g}^{2}}{n_{2}^{2}+n_{g}^{2}}}
$$

Furthermore, the total internal reflection (TIR) occurs when the light waves travel from a material with a higher refractive index to another material with a lower refractive index. For TIR, the incident angle $(\theta)$ must be greater than the critical angle $\left(\theta_{c}\right)$, therefore $\theta_{c}=\sin ^{-1}\left(n_{2} / n_{1}\right)$. Likewise, the evanescent waves induced within the medium with a lower refractive index, the induced waves decay exponentially to the interface distance between both mediums. Therefore, the evanescent wave vector (Kevan. II) can be determined by Equation (15).

$$
\begin{gathered}
k_{\text {evan. } \|}=\frac{2 \pi}{\lambda} n_{1} \sin (\theta) \\
k_{\text {evan. } .}=K_{S P}
\end{gathered}
$$

where $\theta, n_{1}$, and $\lambda$ are the incident angle, the refractive index of gold, and the wavelength of the incident light, respectively. When the evanescent wave excites the surface plasmons, the intensity of reflected light decreases sharply. The decrease in excited plasmons induces the energy conversion to 
photons and phonons. Therefore, the $K_{S P}$ should be equal to $K_{\text {evan. } \|}$. According to the requirement of the phase-matching condition for SPR excitations [68]; $\theta_{S P R}$ angle as a function of related refractive index could be obtained by solving Equations (14)-(16) together (Equation (17)).

$$
\theta_{s p r}=\sin ^{-1}\left(\frac{2 \pi}{n_{1}} \sqrt{\frac{n_{2} n_{g}}{n_{2}+n_{g}}}\right)
$$

Specifically, the SPR angle of the incident P-polarized light mainly depends on $n_{1}, n_{2}$, and $n_{g}$. The modification in the refractive index of the second medium near the dielectric and metal interface and the change in resonance angle also take place, accordingly. Hence, the shift in the $\theta_{S P R}$ angle could be used to assess the variation induced by the change in the refractive index of plasmonic material. Based on the above equations, the SPR reflectivity curve was also simulated using the in-house developed MATLAB simulation program. The program allows the flexible designing of the Kretschmann SPR configuration based on the values of the optical constants and thicknesses of the material of interest, as shown in Figure $7 \mathrm{~b}$. The improved SPR response of the B-rGO samples was investigated by comparing the detection accuracy value or the signal to noise ratio (SNR) of the bare gold, GO, and B-doped GO. The sensitivity and the SNR were calculated using Equation (18) [2], where SNR is explained in terms of FWHM.

$$
S N R=\frac{\Delta \theta_{s p r}}{F W H M}
$$

where, $\triangle \theta_{S P R}$ and FWHM are the SPR angle shift and the full-width-at-half maximum, respectively. Besides, the penetration depth through the dielectric medium $\left(\delta_{d}\right)$ were also considered as an essential parameter, which describes the length over which the surface plasmon was sensitive to the changes in the refractive index in the dielectric medium. It could be calculated using Equation (19).

$$
\delta_{d}=\frac{\lambda_{0}}{2 \pi}\left[\frac{\varepsilon_{m}+\varepsilon_{d}}{\left(\varepsilon_{d}\right)}\right]^{1 / 2}
$$

where, $\lambda_{0}$ is the free space wavelength, $\varepsilon_{m}$, and $\varepsilon_{d}$ are the real part dielectric constants of gold and the adjacent material to the gold, correspondingly [2].

\subsection{Fabrication of $A u / B-r G O$ Sensor Chips}

The gold-coated glass (AU. 0500. ALSI, City, Fitchburg, WI, USA) with a uniform thickness of $50 \mathrm{~nm}$ Au layer was purchased from Platypus Technology, USA. The thickness of the Au layer was further confirmed by the surface roughness tester (SV 3000, Mitutoyo, Aurora, IL, USA). The B-rGO dispersion solution $(0.25 \mathrm{mg} / \mathrm{mL})$ in NMP was attained after the $1 \mathrm{~h}$ sonication process, using the probe sonicator. After that, the unexploited flakes of B-rGO were removed by the centrifuge process at $6000 \mathrm{rpm}$ for 10 minutes. The Au-coated glass was washed with DI water before the deposition of the B-rGO layer. The uniform B-rGO thin film was attained by using a spin-coating technique at the rotation speed of 4,500 rpm followed by its thickness measurement using the surface roughness tester. Later, the sensing layer coated on top of the gold film was attached to the SF11 prism using the Norland index matching liquid, Newport to prepare the sensor chip.

\section{Conclusions}

In this work, we studied the enhanced SPR response with a tunable optical bandgap in the visible region of B-rGO. The B-rGO was synthesized using a customized microwave-assisted hydrothermal approach. The successful synthesis of B-rGO was confirmed by standard FESEM, FTIR, RAMAN, XRD, and XPS-based analyses. The optical bandgap and optical constants for the B-rGO were extracted from the UV-Vis absorption spectrum. The direct bandgap for the three different B-rGO samples was 
found to be $\sim 2.91,2.94$, and $3.05 \mathrm{eV}$, whereas the optical bandgap for $\mathrm{GO}$ was found to be $3.41 \mathrm{eV}$. Interestingly, the enhanced P-type electrical conductivity with reduced optical bandgap was also observed. The surface plasmon resonance-based sensing response of the samples was investigated theoretically as well as experimentally using the customized Kretschmann configuration and found to be enhanced for B-rGO. The acquired results validate the tunability of the optical bandgap and the enhanced surface plasmons resonance in B-rGO. The comparative depth penetration response obtained for B-rGO samples, predicts its utilization in future highly sensitive SPR-based sensors. The tunable optical and electronic properties of B-rGO could be further optimized for optoelectronic, sensor, and light-harvesting applications.

Supplementary Materials: The Supplementary Materials are available online.

Author Contributions: M.J.: Conceptualization, Data Curation, Formal Analysis, Writing-Original Draft. M.H.M.K.: Conceptualization, Funding Acquisition, Project Administration, Supervision. G.W.: Funding Acquisition, Conceptualization, Project Administration, And Supervision. N.T.: Data Curation and Validation. M.S.M.S.: Resources. Z.U.: Visualization, Review \& Editing. P.K.: Formal Analysis and Review \& Editing. A.Y.: Methodology and Experiment Design. F.U.: Software and Development and Design of Methodology. All authors have read and agreed to the published version of the manuscript.

Funding: This research was funded by Yayasan Universiti Teknologi PETRONAS (YUTP), under grant number 015LC0-010. The efforts at Lehigh (N.T.) was supported by the U.S. National Science Foundation (DMR 1726395), and the Daniel E. '39 and Patricia M. Smith Endowed Chair Professorship Fund.

Acknowledgments: This work is part of the "Investigation and Fabrication of Modified Graphene Active Layers In Heterostructure Light-Emitting Device For Transportation And Oil \& Gas Industries" Project. We would like to acknowledge the Universiti Teknologi PETRONAS and Department of Electronic Engineering, BUITEMS, 87300 Quetta, Balochistan, Pakistan for collaboration and technical support. We also acknowledge the Lehigh University, the USA for improving and giving technical support on this article.

Conflicts of Interest: The authors declare no conflict of interest.

\section{References}

1. Prabowo, B.A.; Purwidyantri, A.; Liu, K.C. Surface plasmon resonance optical sensor: A review on light source technology. Biosensors 2018, 8, 80. [CrossRef] [PubMed]

2. Santos, J.L.; Farahi, F. Handbook of Optical Sensors; CRC Press: Boca Raton, FL, USA, 2014.

3. Ullah, Z.; Witjaksono, G.; Nawi, I.; Tansu, N.; Khattak, M.I.; Junaid, M. A review on the development of tunable graphene nanoantennas for terahertz optoelectronic and plasmonic applications. Sensors 2020, 20, 1401. [CrossRef] [PubMed]

4. Ullah, Z.; Nawi, I.; Witjaksono, G.; Tansu, N.; Khattak, M.I.; Junaid, M.; Siddiqui, M.A.; Magsi, S.A. Dynamic absorption enhancement and equivalent resonant circuit modeling of tunable graphene-metal hybrid antenna. Sensors 2020, 20, 3187. [CrossRef]

5. Schwank, J. Catalytic gold-Applications of elemental gold in heterogeneous catalysis. Gold Bull. 1983, 16, 103-110. [CrossRef]

6. Wu, L.; Chu, H.S.; Koh, W.S.; Li, E.P. Highly sensitive graphene biosensors based on surface plasmon resonance. Opt. Express 2010, 18, 14395. [CrossRef]

7. Parsons, R.; Ritzoulis, G. Adsorption on stepped surfaces of platinum and gold single crystals. J. Electroanal. Chem. 1991, 318, 1-24. [CrossRef]

8. Lin, Z.D.; Hsiao, C.H.; Young, S.J.; Huang, C.S.; Chang, S.J.; Wang, S.B. Carbon nanotubes with adsorbed Au for sensing gas. IEEE Sens. J. 2013, 13, 2423-2427. [CrossRef]

9. Cittadini, M.; Bersani, M.; Perrozzi, F.; Ottaviano, L.; Wlodarski, W.; Martucci, A. Graphene oxide coupled with gold nanoparticles for localized surface plasmon resonance based gas sensor. Carbon N. Y. 2014, 69, 452-459. [CrossRef]

10. Wei, W.; Nong, J.; Zhang, G.; Tang, L.; Jiang, X.; Chen, N.; Luo, S.; Lan, G.; Zhu, Y. Graphene-based long-period fiber grating surface plasmon resonance sensor for high-sensitivity gas sensing. Sensors 2017, 17, 2. [CrossRef] 
11. Omar, N.A.S.; Fen, Y.W.; Saleviter, S.; Daniyal, W.M.E.M.M.; Anas, N.A.A.; Ramdzan, N.S.M.; Roshidi, M.D.A. Development of a graphene-based surface plasmon resonance optical sensor chip for potential biomedical application. Materials 2019, 12, 1928. [CrossRef]

12. Kamaruddin, N.H.; Bakar, A.A.A.; Mobarak, N.N.; Dzulkefly Zan, M.S.; Arsad, N. Binding affinity of a highly sensitive $\mathrm{Au} / \mathrm{Ag} / \mathrm{Au} / \mathrm{Chitosan}$-graphene oxide sensor based on direct detection of $\mathrm{Pb} 2+$ and $\mathrm{Hg} 2+$ ions. Sensors 2017, 17, 2277. [CrossRef] [PubMed]

13. Usman, F.; Dennis, J.O.; Seong, K.C.; Ahmed, A.Y.; Ferrell, T.L.; Fen, Y.W.; Sadrolhosseini, A.R.; Ayodele, O.B.; Meriaudeau, F.; Saidu, A. Enhanced Sensitivity of Surface Plasmon Resonance Biosensor Functionalized with Doped Polyaniline Composites for the Detection of Low-Concentration Acetone Vapour. J. Sensors 2019, 2019. [CrossRef]

14. Xue, T.; Yu, S.; Zhang, X.; Zhang, X.; Wang, L.; Bao, Q.; Chen, C.; Zheng, W.; Cui, X. R6G molecule induced modulation of the optical properties of reduced graphene oxide nanosheets for use in ultrasensitive SPR sensing. Sci. Rep. 2016, 6. [CrossRef]

15. Stroe, M.; Cristea, M.; Matei, E.; Galatanu, A.; Cotet, L.C.; Pop, L.C.; Baia, M.; Danciu, V.; Anghel, I.; Baia, L.; et al. Optical properties of composites based on graphene oxide and polystyrene. Molecules 2020, 25, 2419. [CrossRef]

16. Jewel, M.U.; Monne, M.A.; Mishra, B.; Chen, M.Y. Inkjet-printed molybdenum disulfide and nitrogen-doped graphene active layer high on/off ratio transistors. Molecules 2020, 25, 1081. [CrossRef]

17. Wang, J.; Chen, Y.; Yuan, L.; Zhang, M.; Zhang, C. Scandium decoration of boron doped porous graphene for high-capacity hydrogen storage. Molecules 2019, 24, 2382. [CrossRef] [PubMed]

18. Thirumal, V.; Pandurangan, A.; Jayavel, R.; Ilangovan, R. Synthesis and characterization of boron doped graphene nanosheets for supercapacitor applications. Synth. Met. 2016, 220, 524-532. [CrossRef]

19. Sahoo, M.; Sreena, K.P.; Vinayan, B.P.; Ramaprabhu, S. Green synthesis of boron doped graphene and its application as high performance anode material in Li ion battery. Mater. Res. Bull. 2015, 61, 383-390. [CrossRef]

20. Vijaya, P.M.; Kumar, M.P.; Takahashi, C.; Kundu, S.; Narayanan, T.N.; Pattanayak, D.K. Boron-doped graphene quantum dots: An efficient photoanode for a dye sensitized solar cell. New J. Chem. 2019, 43, 14313-14319.

21. Tang, Z.R.; Zhang, Y.; Zhang, N.; Xu, Y.J. New insight into the enhanced visible light photocatalytic activity over boron-doped reduced graphene oxide. Nanoscale 2015, 7, 7030-7034. [CrossRef]

22. Lin, T.; Huang, F.; Liang, J.; Wang, Y. A facile preparation route for boron-doped graphene, and its CdTe solar cell application. Energy Environ. Sci. 2011, 4, 862-865. [CrossRef]

23. Panchakarla, L.S.; Subrahmanyam, K.S.; Saha, S.K.; Govindaraj, A.; Krishnamurthy, H.R.; Waghmare, U.V.; Rao, C.N.R. Synthesis, structure, and properties of boron- and nitrogen-doped graphene. Adv. Mater. 2009, 21, 4726-4730. [CrossRef]

24. Wu, T.; Shen, H.; Sun, L.; Cheng, B.; Liu, B.; Shen, J. Nitrogen and boron doped monolayer graphene by chemical vapor deposition using polystyrene, urea and boric acid. New J. Chem. 2012, 36, 1385-1391. [CrossRef]

25. Liu, H.; Liu, Y.; Zhu, D. Chemical doping of graphene. J. Mater. Chem. 2011, 21, 3335-3345. [CrossRef]

26. Le, G.T.T.; Manyam, J.; Opaprakasit, P.; Chanlek, N.; Grisdanurak, N.; Sreearunothai, P. Divergent mechanisms for thermal reduction of graphene oxide and their highly different ion affinities. Diam. Relat. Mater. 2018, 89, 246-256. [CrossRef]

27. Umrao, S.; Gupta, T.K.; Kumar, S.; Singh, V.K.; Sultania, M.K.; Jung, J.H.; Oh, I.K.; Srivastava, A. Microwave-Assisted Synthesis of Boron and Nitrogen co-doped Reduced Graphene Oxide for the Protection of Electromagnetic Radiation in Ku-Band. ACS Appl. Mater. Interfaces 2015, 7, 19831-19842. [CrossRef] [PubMed]

28. Ci, L.; Song, L.; Jin, C.; Jariwala, D.; Wu, D.; Li, Y.; Srivastava, A.; Wang, Z.F.; Storr, K.; Balicas, L.; et al. Atomic layers of hybridized boron nitride and graphene domains. Nat. Mater. 2010, 9, 430-435. [CrossRef] [PubMed]

29. Huang, Y.; Zhu, C.; Pan, H.; Xu, D.; Lu, T.; Mao, L.; Meng, X.; Chen, Z.; Zhang, D.; Zhu, S. Fabrication of $\mathrm{AgBr} /$ boron-doped reduced graphene oxide aerogels for photocatalytic removal of $\mathrm{Cr}(\mathrm{VI})$ in water. RSC Adv. 2017, 7, 36000-36006. [CrossRef]

30. Hontoria-Lucas, C.; López-Peinado, A.J.; López-González, J.d.D.; Rojas-Cervantes, M.L.; Martín-Aranda, R.M. Study of oxygen-containing groups in a series of graphite oxides: Physical and chemical characterization. Carbon N. Y. 1995, 33, 1585-1592. [CrossRef]

31. Acik, M.; Lee, G.; Mattevi, C.; Pirkle, A.; Wallace, R.M.; Chhowalla, M.; Cho, K.; Chabal, Y. The role of oxygen during thermal reduction of graphene oxide studied by infrared absorption spectroscopy. J. Phys. Chem. C 2011, 115, 19761-19781. [CrossRef] 
32. Page, A.J.; Chou, C.P.; Pham, B.Q.; Witek, H.A.; Irle, S.; Morokuma, K. Quantum chemical investigation of epoxide and ether groups in graphene oxide and their vibrational spectra. Phys. Chem. Chem. Phys. 2013, 15, 3725-3735. [CrossRef] [PubMed]

33. Das, A.K.; Srivastav, M.; Layek, R.K.; Uddin, M.E.; Jung, D.; Kim, N.H.; Lee, J.H. Iodide-mediated room temperature reduction of graphene oxide: A rapid chemical route for the synthesis of a bifunctional electrocatalyst. J. Mater. Chem. A 2014, 2, 1332-1340. [CrossRef]

34. Xu, Y.; Bai, H.; Lu, G.; Li, C.; Shi, G. Flexible graphene films via the filtration of water-soluble noncovalent functionalized graphene sheets. J. Am. Chem. Soc. 2008, 130, 5856-5857. [CrossRef] [PubMed]

35. Van Khai, T.; Na, H.G.; Kwak, D.S.; Kwon, Y.J.; Ham, H.; Shim, K.B.; Kim, H.W. Comparison study of structural and optical properties of boron-doped and undoped graphene oxide films. Chem. Eng. J. 2012, 211-212, 369-377. [CrossRef]

36. Rodríguez, M.G.; Kharissova, O.V.; Ortiz-Méndez, U. Formation of boron carbide nanofibers and nanobelts from heated by microwave. Rev. Adv. Mater. Sci. 2004, 7, 55-60.

37. Shirai, K.; Emura, S.; Gonda, S.I.; Kumashiro, Y. Infrared study of amorphous B1-xCx films. J. Appl. Phys. 1995, 78, 3392-3400. [CrossRef]

38. Li, R.; Wei, Z.; Gou, X.; Xu, W. Phosphorus-doped graphene nanosheets as efficient metal-free oxygen reduction electrocatalysts. RSC Adv. 2013, 3, 9978-9984. [CrossRef]

39. Yuan, B.; Xing, W.; Hu, Y.; Mu, X.; Wang, J.; Tai, Q.; Li, G.; Liu, L.; Liew, K.M.; Hu, Y. Boron/phosphorus doping for retarding the oxidation of reduced graphene oxide. Carbon N. Y. 2016, 101, 152-158. [CrossRef]

40. Xue, Y.; Yu, D.; Dai, L.; Wang, R.; Li, D.; Roy, A.; Lu, F.; Chen, H.; Liu, Y.; Qu, J. Three-dimensional B,N-doped graphene foam as a metal-free catalyst for oxygen reduction reaction. Phys. Chem. Chem. Phys. 2013, 15, 12220-12226. [CrossRef] [PubMed]

41. Nozaki, H.; Itoh, S. Structural stability of BC2N. J. Phys. Chem. Solids 1996, 57, 41-49. [CrossRef]

42. Yeom, D.Y.; Jeon, W.; Tu, N.D.K.; Yeo, S.Y.; Lee, S.S.; Sung, B.J.; Chang, H.; Lim, J.A.; Kim, H. High-concentration boron doping of graphene nanoplatelets by simple thermal annealing and their supercapacitive properties. Sci. Rep. 2015, 5. [CrossRef] [PubMed]

43. Oh, Y.J.; Yoo, J.J.; Kim, Y.I.; Yoon, J.K.; Yoon, H.N.; Kim, J.H.; Park, S. Bin Oxygen functional groups and electrochemical capacitive behavior of incompletely reduced graphene oxides as a thin-film electrode of supercapacitor. Electrochim. Acta 2014, 116, 118-128. [CrossRef]

44. Mokhtar Mohamed, M.; Mousa, M.A.; Khairy, M.; Amer, A.A. Nitrogen Graphene: A New and Exciting Generation of Visible Light Driven Photocatalyst and Energy Storage Application. ACS Omega 2018, 3, 1801-1814. [CrossRef] [PubMed]

45. Susanti, D.; Andrameda, Y.A.; Nurdiansah, H.; Purwaningsih, H.; Pradesar, Y.; Ardhyananta, H. The Preparation of Electrical Double Layer Capacitor (EDLC) from Boron-doped Reduced-Graphene Oxide (B-rGO) Material. IOP Conf. Ser. Mater. Sci. Eng. 2019, 547. [CrossRef]

46. Rao, C.N.R.; Panchakarla, L.S.; Govindaraj, A.; Rao, C.N.R. Nitrogen- and Boron-Doped Double-Walled Carbon Nanotubes. Trends Chem. Mater. 2008, 552-558.

47. Cermignani, W.; Paulson, T.E.; Onneby, C.; Pantano, C.G. Synthesis and characterization of boron-doped carbons. Carbon N. Y. 1995, 33, 367-374. [CrossRef]

48. Jeong, H.K.; Yun, P.L.; Lahaye, R.J.W.E.; Park, M.H.; Kay, H.A.; Ick, J.K.; Yang, C.W.; Chong, Y.P.; Ruoff, R.S.; Young, H.L. Evidence of graphitic AB stacking order of graphite oxides. J. Am. Chem. Soc. 2008, 130, 1362-1366. [CrossRef]

49. Sahu, R.S.; Bindumadhavan, K.; Doong, R.-A. Boron-doped reduced graphene oxide-based bimetallic Ni/Fe nanohybrids for the rapid dechlorination of trichloroethylene. Environ. Sci. Nano 2017, 4, 565-576. [CrossRef]

50. Thomas, D.-G.; Kavak, E.; Hashemi, N.; Montazami, R.; Hashemi, N. Synthesis of Graphene Nanosheets through Spontaneous Sodiation Process. C-J. Carbon Res. 2018, 4, 42. [CrossRef]

51. Zhao, B.; Liu, P.; Jiang, Y.; Pan, D.; Tao, H.; Song, J.; Fang, T.; Xu, W. Supercapacitor performances of thermally reduced graphene oxide. J. Power Sources 2012, 198, 423-427. [CrossRef]

52. Pham, V.H.; Pham, H.D.; Dang, T.T.; Hur, S.H.; Kim, E.J.; Kong, B.S.; Kim, S.; Chung, J.S. Chemical reduction of an aqueous suspension of graphene oxide by nascent hydrogen. J. Mater. Chem. 2012, 22, 10530-10536. [CrossRef]

53. InstaMan UV Vis Spectroscopy Band Gap Calculation. Available online: https://instanano.com/ characterization/theoretical/uv-vis-spectroscopy-band-gap-calculation/ (accessed on 13 May 2020). 
54. Habte, A.T.; Ayele, D.W.; Hu, M. Synthesis and Characterization of Reduced Graphene Oxide (rGO) Started from Graphene Oxide (GO) Using the Tour Method with Different Parameters. Adv. Mater. Sci. Eng. 2019, 2019. [CrossRef]

55. Marschall, R.; Wang, L. Non-metal doping of transition metal oxides for visible-light photocatalysis. Catal. Today 2014, 225, 111-135. [CrossRef]

56. Handbook of Surface Plasmon Resonance; Schasfoort, R.B.M. (Ed.) The Royal Society of Chemistry: London, UK, 2017; ISBN 978-1-78262-730-2.

57. Johnson, P.B.; Christy, R.W. Optical constants of the noble metals. Phys. Rev. B 1972, 6, 4370-4379. [CrossRef]

58. Schmiedova, V.; Pospisil, J.; Kovalenko, A.; Ashcheulov, P.; Fekete, L.; Cubon, T.; Kotrusz, P.; Zmeskal, O.; Weiter, M. Physical Properties Investigation of Reduced Graphene Oxide Thin Films Prepared by Material Inkjet Printing. J. Nanomater. 2017, 2017. [CrossRef]

59. Knoll, W. Interfaces and Thin Films As Seen By Bound Electromagnetic Waves. Annu. Rev. Phys. Chem. 1998, 49, 569-638. [CrossRef]

60. Yar, A.; Dennis, J.O.; Mohamed Saheed, M.S.; Mohamed, N.M.; Irshad, M.I.; Mumtaz, A.; Jose, R. Physical reduction of graphene oxide for supercapacitive charge storage. J. Alloys Compd. 2020, 822. [CrossRef]

61. Perkampus, H.-H. UV-VIS Spectroscopy and Its Applications; Springer: Berlin/Heidelberg, Germany, 1992.

62. Nic, M.; Jirat, J.; Kosata, B. Compendium of Chemical Terminology; Blackwell Scientific Publications: Hoboken, NJ, USA, 2012.

63. Aziz, S.B.; Hassan, A.Q.; Mohammed, S.J.; Karim, W.O.; Kadir, M.F.Z.; Tajuddin, H.A.; Chan, N.N.M.Y. Structural and optical characteristics of pva:C-dot composites: Tuning the absorption of ultra violet (uv) region. Nanomaterials 2019, 9, 216. [CrossRef]

64. Tauc, J. Amorphous and Liquid Semiconductors.; Springer Science \& Business Media: Heidelberg, Germany, 1977.

65. Mott, N.F.; Davis, E.A. Conduction In Non-crystalline Systems V. Conductivity, Optical Absorption and Photoconductivity In Amorphous Semiconductors. Philos. Mag. 1970, 22, 903-922.

66. Fan, X.; Shen, Z.; Liu, A.Q.; Kuo, J.L. Band gap opening of graphene by doping small boron nitride domains. Nanoscale 2012, 4, 2157-2165. [CrossRef]

67. Matsubara, K.; Kawata, S.; Minami, S. Optical chemical sensor based on surface plasmon measurement. Appl. Opt. 1988, 27, 1160. [CrossRef] [PubMed]

68. Piliarik, M.; Homola, J. Surface plasmon resonance (SPR) sensors: Approaching their limits? Opt. Express 2009, 17, 16505. [CrossRef] [PubMed]

Sample Availability: Samples of the compounds are not available from the authors.

(C) 2020 by the authors. Licensee MDPI, Basel, Switzerland. This article is an open access article distributed under the terms and conditions of the Creative Commons Attribution (CC BY) license (http://creativecommons.org/licenses/by/4.0/). 